\title{
How the dark energy can reconcile Planck with local determination of the Hubble constant
}

\author{
Qing-Guo Huang ${ }^{1,2, a}$, Ke Wang ${ }^{1, b}$ \\ ${ }^{1}$ CAS Key Laboratory of Theoretical Physics, Institute of Theoretical Physics, Chinese Academy of Sciences, Beijing 100190, China \\ ${ }^{2}$ School of Physical Sciences, University of Chinese Academy of Sciences, No. 19A Yuquan Road, Beijing 100049, China
}

Received: 28 June 2016 / Accepted: 5 September 2016 / Published online: 16 September 2016

(C) The Author(s) 2016. This article is published with open access at Springerlink.com

\begin{abstract}
We try to reconcile the tension between the local $2.4 \%$ determination of Hubble constant and its global determination by Planck CMB data and BAO data through modeling the dark energy variously. We find that the chi-square is significantly reduced by $\Delta \chi_{\text {all }}^{2}=-6.76$ in the redshiftbinned dark energy model where the $68 \%$ limits of the equation of state of dark energy read $w(0 \leq z \leq 0.1)=$ $-1.958_{-0.508}^{+0.509}, w(0.1<z \leq 1.5)=-1.006_{-0.082}^{+0.092}$, and here $w(z>1.5)$ is fixed to -1 .
\end{abstract}

\section{Introduction}

Recently Riess et al. in [1] confirmed and improved their former determination of the Hubble constant $\left(H_{0}=73.8 \pm\right.$ $\left.2.4 \mathrm{~km} \mathrm{~s}^{-1} \mathrm{Mpc}^{-1}[2]\right)$ :

$H_{0}=73.00 \pm 1.75 \mathrm{~km} \mathrm{~s}^{-1} \mathrm{Mpc}^{-1}$

at $68 \%$ confidence level (CL) by still using three distance anchors (Milky Way (MW), Large Magellic Cloud (LMC) and NGC 4258). The improvement in uncertainty is due to not only an enlarged number of SN hosts, Cepheids (in the LMC), and MW Cepheids (with HST-based trigonometric parallaxes) but also a revised distance to NGC 4258 and the LMC. In 2013 Efstathiou revisited the dataset of [2] and yielded another two different value of $H_{0}: H_{0}=$ $72.5 \pm 2.5 \mathrm{~km} \mathrm{~s}^{-1} \mathrm{Mpc}^{-1}$ using three anchors (MW, LMC and NGC 4258) and $H_{0}=70.6 \pm 3.3 \mathrm{~km} \mathrm{~s}^{-1} \mathrm{Mpc}^{-1}$ with NGC 4258 as only anchor in [3]. As emphasized in [1], although these three local determinations of $H_{0}$ with the same three anchors are consistent with each other, $H_{0}=$ $73.00 \pm 1.75 \mathrm{~km} \mathrm{~s}^{-1} \mathrm{Mpc}^{-1}$ is considered to be the best one (R16, hereafter) due to the aforementioned improvement.

\footnotetext{
a e-mail: huangqg@itp.ac.cn

be-mail: wangke@itp.ac.cn
}

On the other hand, there are some widely accepted global determinations of $H_{0}$ mainly derived from $\mathrm{CMB}$ data under the assumption of the $\Lambda \mathrm{CDM}$ model: $H_{0}=$ $69.7 \pm 2.1 \mathrm{~km} \mathrm{~s}^{-1} \mathrm{Mpc}^{-1}$ from WMAP9 [4]; ${ }^{1} H_{0}=$ $68.0 \pm 0.7 \mathrm{~km} \mathrm{~s}^{-1} \mathrm{Mpc}^{-1}$ from WMAP9 + BAO [4]; $H_{0}=69.3 \pm 0.7 \mathrm{~km} \mathrm{~s}^{-1} \mathrm{Mpc}^{-1}$ from WMAP9 + ACT + SPT $+\mathrm{BAO}[7] ; H_{0}=67.3 \pm 1.0 \mathrm{~km} \mathrm{~s}^{-1} \mathrm{Mpc}^{-1}$ from Planck TT + lowP [4]; $H_{0}=67.6 \pm 0.6 \mathrm{~km} \mathrm{~s}^{-1} \mathrm{Mpc}^{-1}$ from Planck $\mathrm{TT}+$ lowP + BAO [4]. In addition, in [8] combining the low-redshift and high-redshift isotropic BAO data, the Hubble constant was given by $H_{0}=68.17_{-1.56}^{+1.55} \mathrm{~km} \mathrm{~s}^{-1} \mathrm{Mpc}^{-1}$ which is consistent with the results from CMB data. Comparing R16 with these cosmological estimates, we find that there is a longstanding tension which becomes more significant now.

Since the global determinations of $H_{0}$ are highly modeldependent, there is a well-known solution to this tension: adding additional dark radiation to the base $\Lambda \mathrm{CDM}$ model [1,4,9-11]. Recently in [12] the authors found that a variation of equation of state (EOS) of Dark Energy (DE) in a 12parameter extension of the $\Lambda \mathrm{CDM}$ model is more favored than adding dark radiation. Furthermore, they pointed out that the tension between R16 and the combination of Planck 2015 data and BAO data still exists in their 12-parameter extension. In this short paper we try to reconcile the tension between R16 with the combination of Planck CMB data and BAO data through modeling the DE variously.

In the literature there are several well-known DE models: the $\triangle \mathrm{CDM}$ model where $\mathrm{DE}$ is described by a cosmological constant with EOS fixed as -1 ; the $w$ CDM model where DE has a constant EOS $w$; the CPL model in which EOS of DE is time-dependent $[13,14]$. In general the non-parametric reconstructions of the EOS of $\mathrm{DE}$ in bins of redshift, like those in [15-17], are considered to be model-independent.

\footnotetext{
1 The constraint from WMAP9 is different the original ones in $[5,6]$ which does not include $0.06 \mathrm{eV}$ neutrino mass.
} 
We will investigate whether these DE models can be used to reconcile the tension on the determinations of Hubble constant in this short paper.

The rest of the paper is arranged as follows. In Sect. 2, we reveal our methodology and cosmological datasets used in this paper. In Sect. 3, we globally fit all of the cosmological parameters in various extensions to the $\Lambda \mathrm{CDM}$ model by combining R16, Planck and BAO datasets. A brief summary and discussion are included in Sect. 4.

\section{Data and methodology}

In this paper, we try to reconcile the tension between R16 and the global determination of $H_{0}$ by $\mathrm{CMB}$ data released by Planck collaboration in 2015 and BAO measurements. Here we add the R16 prior to the data combination of Planck 2015 data (Planck TT, TE, EE + lowP + lensing) [4] and the BAO data including 6dFGS $\left(z_{\text {eff }}=0.106\right)$ [18], MGS $\left(z_{\text {eff }}=0.15\right)$ [19], BOSS DR12 LOWZ $\left(z_{\text {eff }}=0.32\right)$ [20] and CMASS ( $\left.z_{\text {eff }}=0.57\right)[20]$.

We focus on a spatially flat universe and the Friedmann equation reads

$H^{2}=\frac{8 \pi G}{3}\left[\rho_{r}(0)(1+z)^{4}+\rho_{m}(0)(1+z)^{3}+\rho_{d e}(z)\right]$,

where the energy density of DE is related to its $\operatorname{EOS}(w(z))$ by

$\rho_{d e}(z)=\rho_{d e}(0) \exp \left\{3 \int_{0}^{z} \frac{\mathrm{d} z^{\prime}}{\left(1+z^{\prime}\right)}\left[1+w\left(z^{\prime}\right)\right]\right\}$.

The global determination of $H_{0}$ highly depends on the evolution of the energy density at low redshift, and consequently on the evolution of EOS of DE at low redshift.

In the $\Lambda \mathrm{CDM}$ model, $w(z)=-1$ and there are six base cosmological parameters which are denoted by $\left\{\Omega_{b} h^{2}, \Omega_{c} h^{2}\right.$, $\left.100 \theta_{\mathrm{MC}}, \tau, n_{s}, \ln \left(10^{10} A_{s}\right)\right\}$. Here $\Omega_{b} h^{2}$ is the physical density of baryons today, $\Omega_{c} h^{2}$ is the physical density of cold dark matter today, $\theta_{\mathrm{MC}}$ is the ratio between the sound horizon and the angular diameter distance at the decoupling epoch, $\tau$ is the Thomson scatter optical depth due to reionization, $n_{s}$ is the scalar spectrum index, and $A_{s}$ is the amplitude of the power spectrum of primordial curvature perturbations at the pivot scale $k_{p}=0.05 \mathrm{Mpc}^{-1}$.

The $w$ CDM model with an arbitrary constant EOS $w$ is the simplest DE extension of the $\triangle \mathrm{CDM}$ model. Therefore, there are six base cosmological parameters plus another free parameter $w$. The CPL model is a widely used DE extension of the $\Lambda \mathrm{CDM}$ model and the EOS of DE is parametrized by

$w(z)=w_{0}+w_{a} \frac{z}{1+z}$, where $w_{0}$ and $w_{a}$ are two free parameters in addition to the six base free parameters mentioned above. Usually we use $w_{0} w_{a}$ CDM to donate this model.

Furthermore, we also consider two model-independent redshift-binned DE models. Since the expansion of the universe is insensitive to DE at high redshifts where the energy density of DE becomes negligibly small, we fix the EOS of $\mathrm{DE}$ to be -1 for $z>1.5$ for convenience. The first redshiftbinned DE model is denoted by $w_{0.25} w_{1.5} \mathrm{CDM}$ in which the EOS is divided into three bins as follows:

$w(z)=\left\{\begin{array}{l}w_{0.25}, \quad z \leq 0.25 \\ w_{1.5}, \quad 0.25<z \leq 1.5 \\ -1, \quad z>1.5\end{array}\right.$

where $w_{0.25}$ and $w_{1.5}$ are two free parameters. On the other hand, we notice that the effective redshift for the lowest-redshift $\mathrm{BAO}$ is $z_{\mathrm{eff}}=0.106$. In order to "effectively" exclude the distance scale constraints from BAO datasets, we also consider another redshift-binned DE model $\left(w_{0.1} w_{1.5} \mathrm{CDM}\right.$ model $)$ in which the EOS of DE is given by

$w(z)=\left\{\begin{array}{l}w_{0.1}, \quad z \leq 0.1 \\ w_{1.5}, \quad 0.1<z \leq 1.5 \\ -1, \quad z>1.5\end{array}\right.$

where $w_{0.1}$ and $w_{1.5}$ are two free parameters. In this case, there is no BAO dataset in the first redshift bin $(0 \leq z \leq 0.1)$.

\section{Results}

Our main results are given in Table 1 where we represent the $68 \%$ limits for the cosmological parameters in different DE extensions to $\triangle \mathrm{CDM}$ model from the data combination of Planck TT, TE, EE + lowP + lensing + BAO + R16.

Combining R16 with the Planck 2015 and BAO data, the constraints on the six parameters in the $\Lambda \mathrm{CDM}$ model almost do not change with respect to the case without adding a local $H_{0}$ prior, but $\chi_{H_{0}}^{2}=6.57$ indicates the existence of tension between the local and global determinations of $H_{0}$. For the $\Lambda \mathrm{CDM}$ model, $\chi_{\mathrm{BAO}}^{2}=2.40$ implies that the Planck 2015 and BAO data are consistent with each other.

Compared to the $\Lambda \mathrm{CDM}$ model, the chi-square is reduced by $\Delta \chi_{\text {all }}^{2}=-3.62$ in $w \mathrm{CDM}$ model, and a phantom-like DE with EOS of $w=-1.113_{-0.055}^{+0.056}$ is preferred at more than $95 \%$ CL. However, in the wCDM model, the Hubble constant reads $70.71_{-1.49}^{+1.35} \mathrm{~km} \cdot \mathrm{s}^{-1} \cdot \mathrm{Mpc}^{-1}$, which is still small compared to the local determination.

Even though a parameter $w_{a}$ for describing the evolution of the EOS of DE is included in the $w_{0} w_{a} \mathrm{CDM}$ model, the 
Table 1 The $68 \%$ limits for the cosmological parameters in different DE extensions to $\Lambda$ CDM model from the data combination of Planck TT, $\mathrm{TE}, \mathrm{EE}+$ low $\mathrm{P}+$ lensing $+\mathrm{BAO}+\mathrm{R} 16$. The $\chi^{2}$ for different models against individual data are also listed explicitly

\begin{tabular}{|c|c|c|c|c|c|}
\hline- & $\Lambda \mathrm{CDM}$ & $w \mathrm{CDM}$ & $w_{0} w_{a} \mathrm{CDM}$ & $w_{0.1} w_{1.5} \mathrm{CDM}$ & $w_{0.25} w_{1.5} \mathrm{CDM}$ \\
\hline$\Omega_{b} h^{2}$ & $0.02236_{-0.00015}^{+0.00014}$ & $0.02223_{-0.00016}^{+0.00015}$ & $0.02222 \pm 0.00015$ & $0.02227 \pm 0.00015$ & $0.02225_{-0.00016}^{+0.00015}$ \\
\hline$\Omega_{c} h^{2}$ & $0.1180_{-0.0010}^{+0.0011}$ & $0.1197_{-0.0013}^{+0.0012}$ & $0.1196 \pm 0.0013$ & $0.1191_{-0.0014}^{+0.0013}$ & $0.1193 \pm 0.0013$ \\
\hline $100 \theta_{M C}$ & $1.04101 \pm 0.00029$ & $1.04082_{-0.00030}^{+0.00031}$ & $1.04082_{-0.00032}^{+0.00031}$ & $1.04089 \pm 0.00031$ & $1.04086_{-0.00031}^{+0.00030}$ \\
\hline$\tau$ & $0.071 \pm 0.012$ & $0.057_{-0.014}^{+0.013}$ & $0.058 \pm 0.015$ & $0.064 \pm 0.014$ & $0.061 \pm 0.014$ \\
\hline $\ln \left(10^{10} A_{s}\right)$ & $3.072 \pm 0.023$ & $3.047 \pm 0.025$ & $3.050 \pm 0.028$ & $3.060_{-0.026}^{+0.027}$ & $3.055_{-0.025}^{+0.026}$ \\
\hline$n_{s}$ & $0.9686 \pm 0.0041$ & $0.9643 \pm 0.0044$ & $0.9643_{-0.0044}^{+0.0045}$ & $0.9659 \pm 0.0046$ & $0.9652 \pm 0.0045$ \\
\hline $\operatorname{EOS}(z=0)$ & - & $w=-1.113_{-0.055}^{+0.056}$ & $w_{0}=-1.185_{-0.211}^{+0.185}$ & $w_{0.1}=-1.958_{-0.508}^{+0.509}$ & $w_{0.25}=-1.296_{-0.202}^{+0.203}$ \\
\hline- & - & - & $w_{a}=0.196_{-0.485}^{+0.664}$ & $w_{1.5}=-1.006_{-0.082}^{+0.092}$ & $w_{1.5}=-1.037_{-0.113}^{+0.112}$ \\
\hline$H_{0}\left(\mathrm{~km} \cdot \mathrm{s}^{-1} \cdot \mathrm{Mpc}^{-1}\right)$ & $68.08_{-0.48}^{+0.47}$ & $70.71_{-1.49}^{+1.35}$ & $71.29_{-1.88}^{+1.90}$ & $74.18_{-2.51}^{+2.54}$ & $72.07_{-2.01}^{+1.98}$ \\
\hline$\Omega_{\Lambda}$ & $0.6957 \pm 0.0062$ & $0.7146 \pm 0.0107$ & $0.7190_{-0.0145}^{+0.0163}$ & $0.7411_{-0.0169}^{+0.0199}$ & $0.7256_{-0.0146}^{+0.0171}$ \\
\hline$\Omega_{m}$ & $0.3043 \pm 0.0062$ & $0.2854 \pm 0.0107$ & $0.2810_{-0.0163}^{+0.0145}$ & $0.2589_{-0.0199}^{+0.0169}$ & $0.2744_{-0.0171}^{+0.0146}$ \\
\hline$\chi_{\text {all }}^{2}$ & $12,966.04$ & $12,962.42$ & $12,962.26$ & $12,959.28$ & $12,962.28$ \\
\hline$\chi_{\mathrm{CMB}}^{2}$ & $12,957.07$ & $12,955.64$ & $12,955.50$ & $12,954.74$ & $12,956.44$ \\
\hline$\chi_{\mathrm{BAO}}^{2}$ & 2.40 & 4.38 & 5.10 & 4.48 & 5.24 \\
\hline$\chi_{H_{0}}^{2}$ & 6.57 & 2.40 & 1.66 & 0.06 & 0.60 \\
\hline
\end{tabular}

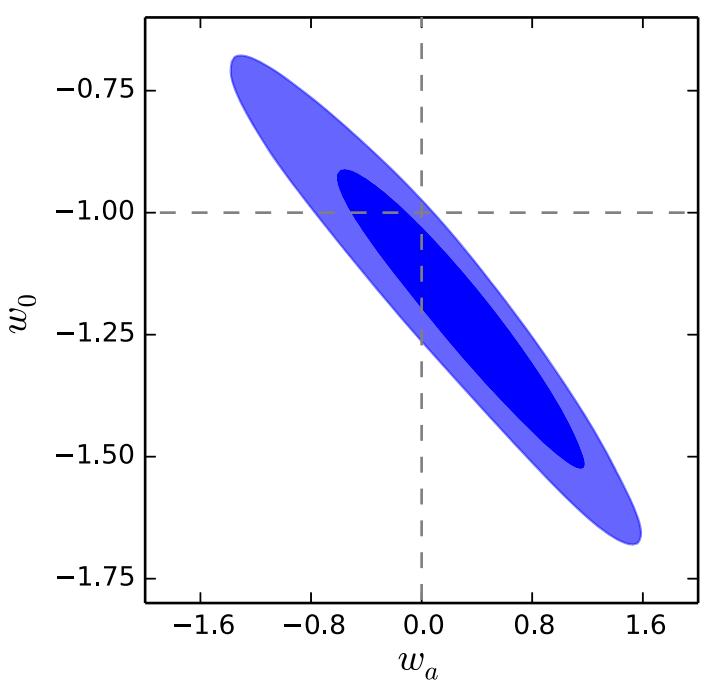

Fig. 1 The contour plot of $w_{0}$ and $w_{a}$ in $w_{0} w_{a}$ CDM model where the EOS is parametrized by $w(z)=w_{0}+w_{a} \frac{z}{1+z}$. The crossing point of gray dashed lines denotes the $\Lambda \mathrm{CDM}$ model

chi-square is almost the same as that in $w \mathrm{CDM}$ model. The contour plot of $w_{0}$ and $w_{a}$ shows up in Fig. 1 .

From Fig. 1, the cosmological constant is disfavored at around $95 \% \mathrm{CL}$, and $w_{0}=-1.185_{-0.211}^{+0.185}$ is consistent with the constraint on $w$ in $w \mathrm{CDM}$ model. Actually, the $w_{0} w_{a} \mathrm{CDM}$ model provides a little bit better fit to the Hubble constant $\left(\chi_{H_{0}}^{2}=1.66\right.$, and $\Delta \chi_{H_{0}}^{2}=-0.74$ compared to $w \mathrm{CDM}$ model), but the fitting to the BAO datasets becomes worse by $\Delta \chi_{\mathrm{BAO}}^{2}=0.72$. Thus, from the statistic point of view, the data do not prefer this model.
It is interesting to see that the chi-square for $w_{0.1} w_{1.5} \mathrm{CDM}$ model is significantly reduced by $\Delta \chi_{\text {all }}^{2}=-6.76 \mathrm{com}$ pared to $\Lambda \mathrm{CDM}$ model, and $\Delta \chi_{\text {all }}^{2}=-3.14$ compared to $w \mathrm{CDM}$ model. Furthermore, $H_{0}=74.18_{-2.51}^{+2.54} \mathrm{~km}$. $\mathrm{s}^{-1} \cdot \mathrm{Mpc}^{-1}$ and $\chi_{H_{0}}^{2}=0.06$ indicate that the global fitting is consistent with the local determination of Hubble constant in $w_{0.1} w_{1.5} \mathrm{CDM}$ model. However, the fitting becomes worse in $w_{0.25} w_{1.5} \mathrm{CDM}$ model $\left(\Delta \chi_{\text {all }}^{2}=3.00\right.$, $\Delta \chi_{\mathrm{CMB}}^{2}=1.70, \Delta \chi_{\mathrm{BAO}}^{2}=0.76$, and $\Delta \chi_{H_{0}}^{2}=0.54 \mathrm{com}$ pared to $w_{0.1} w_{1.5} \mathrm{CDM}$ model). That is to say, all of the individual datasets prefer $w_{0.1} w_{1.5} \mathrm{CDM}$ model compared to $w_{0.25} w_{1.5} \mathrm{CDM}$ model. The plots of EOS at $z=0$ and $w_{1.5}$ in $w_{0.1} w_{1.5} \mathrm{CDM}$ model and $w_{0.25} w_{1.5} \mathrm{CDM}$ model are illustrated in Fig. 2.

We see that the cosmological constant is disfavored at more than $95 \% \mathrm{CL}$ in $w_{0.1} w_{1.5} \mathrm{CDM}$ model. Notice that there are two BAO data points in the range of $0.1<z<$ 0.25 , and they are consistent with Planck CMB data in the $\Lambda \mathrm{CDM}$ model. But the combination of the local determination of Hubble constant and the Planck CMB data prefers a phantom-like DE model. Therefore the EOS of DE $w_{0.25}<-1$ in the range of $0<z<0.25$ enhances the chisquares in $w_{0.25} w_{1.5} \mathrm{CDM}$ model compared to $w_{0.1} w_{1.5} \mathrm{CDM}$ model.

\section{Summary and discussion}

To summarize, the tension between the local determination of Hubble constant and the global determination by Planck 


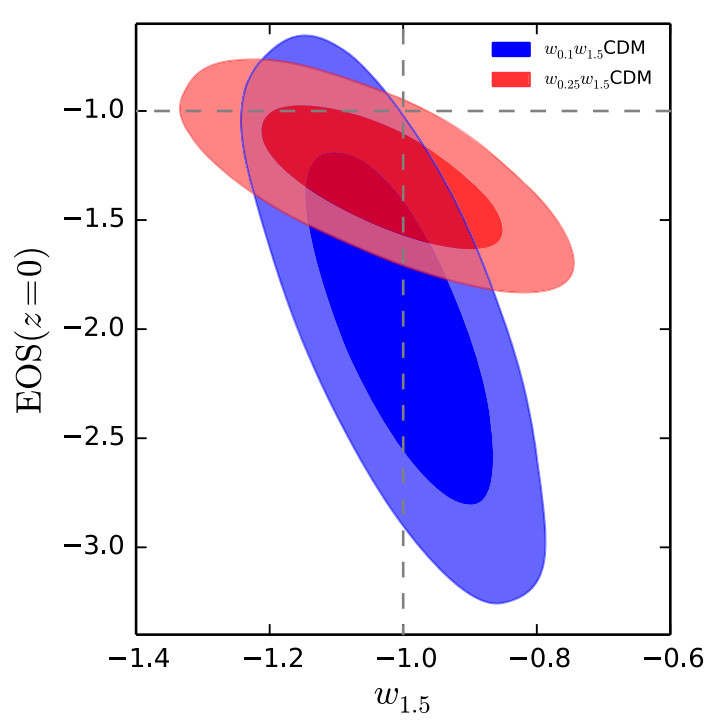

Fig. 2 The plots of EOS at $z=0$ and $w_{1.5}$ in $w_{0.1} w_{1.5} \mathrm{CDM}$ model and $w_{0.25} w_{1.5} \mathrm{CDM}$ model. The crossing point of the two gray dashed lines denotes the $\Lambda \mathrm{CDM}$ model. In particular, the cosmological constant is disfavored at more than $95 \% \mathrm{CL}$ in $w_{0.1} w_{1.5} \mathrm{CDM}$ model which gives a better fit to the data with $\Delta \chi^{2}=-6.76$ compared to the $\Lambda$ CDM model

CMB data and BAO data is statistically significant in the $\Lambda \mathrm{CDM}$ model, and the EOS of DE is preferred to be less than -1 at low redshifts when R16 is added to Planck CMB and $\mathrm{BAO}$ data. The chi-square for the $w_{0.1} w_{0.25} \mathrm{CDM}$ model is significantly reduced by $\Delta \chi_{\text {all }}^{2}=-6.76$ compared to the $\Lambda \mathrm{CDM}$ model, and this model can reconcile the tension on determination of Hubble constant between R16 and the combination of Planck CMB data and BAO data.

Since the EOS of DE is preferred to be less than -1 at low redshifts, the matter energy density today becomes smaller than that in the $\Lambda$ CDM model. See Fig. 3.

In particular, $\Omega_{m}=0.2589_{-0.0199}^{+0.0169}$ in the $w_{0.1} w_{0.25} \mathrm{CDM}$ model, and it is much lower than that in the $\Lambda \mathrm{CDM}$ model $\left(\Omega_{m}=0.3043 \pm 0.0062\right)$. One may worry that this model cannot fit the Planck CMB data because of the lower matter density today. Actually CMB power spectra are more sensitive to $\Omega_{b} h^{2}$ and $\Omega_{c} h^{2}$, not $\Omega_{m}$. The constraints on $\Omega_{b} h^{2}$ and $\Omega_{c} h^{2}$ for different models are showed in Fig. 4, from which we see that the constraints on $\Omega_{b} h^{2}$ and $\Omega_{c} h^{2}$ in all of these models are similar to each other.

Finally, with reduction of large-scale systematic effects in HFI polarization maps, Planck collaboration gave some new constraints on the reinoization optical depth in [21,22] recently. For example, for the likelihood method of Lollipop and the conservative cross-spectra estimators of PCL, the constraint on the reionization optical depth was $\tau=$ $0.053_{-0.016}^{+0.011}$, which is smaller than that given by Planck TT and lowP power spectra. Here the reionization optical depth in the models extended to the $\Lambda \mathrm{CDM}$ models in this paper prefer a lower reionization optical depth which is more con-

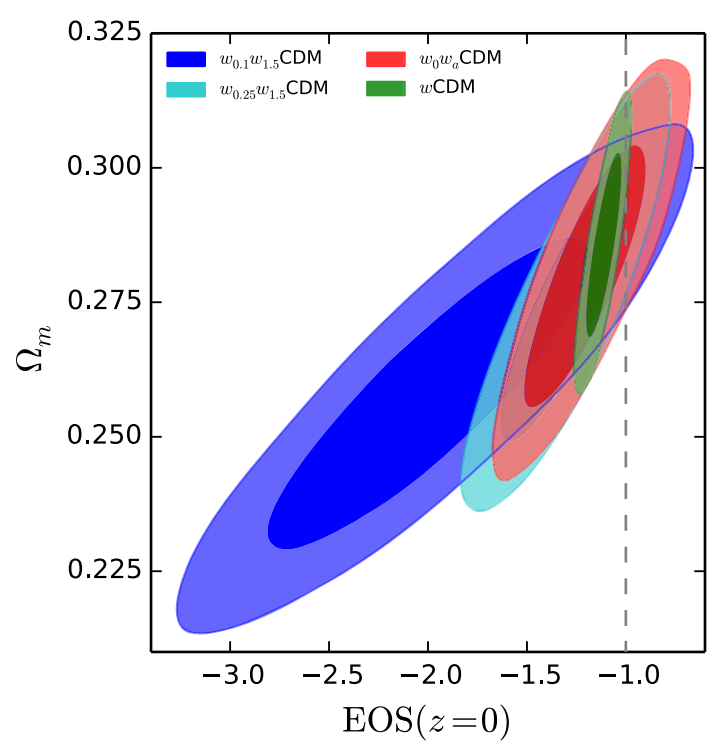

Fig. 3 The contour plot of $\Omega_{m}$ and EOS at $z=0$ for the models extended to the $\Lambda \mathrm{CDM}$ model. The gray dashed line corresponds to $\operatorname{EOS}(z=0)=-1$. Our results indicate that the $\operatorname{EOS}(z=0)<-1$ is preferred in all of the extended models

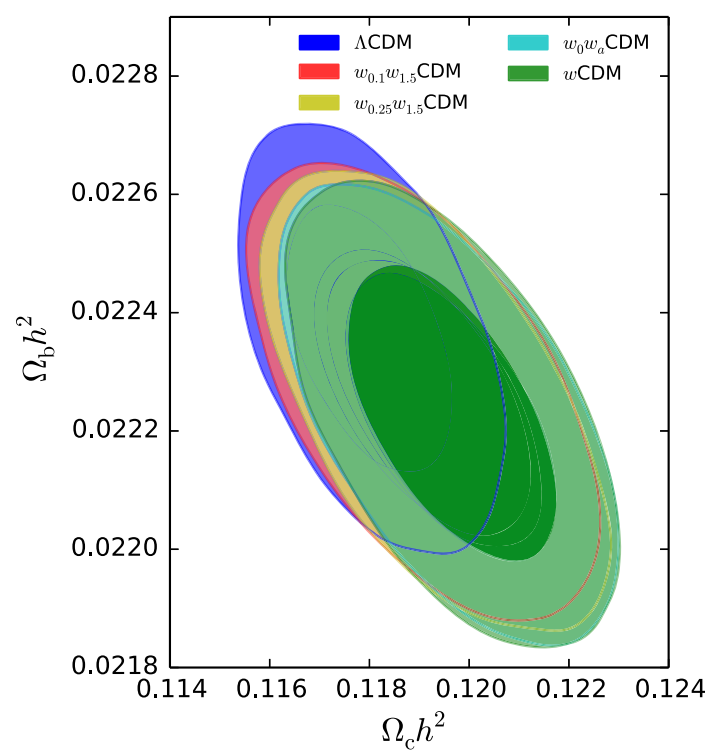

Fig. 4 Constraints on $\Omega_{b} h^{2}$ and $\Omega_{c} h^{2}$ for different DE extension of the $\Lambda \mathrm{CDM}$ model. Even though the values of $\Omega_{m}$ in different models looks different, $\Omega_{b} h^{2}$ and $\Omega_{c} h^{2}$ are coincided with each other

sistent with the recent result released by the Planck collaboration.

Even though a very rare fluctuation might explain the tension between the local determination and the global determination of Hubble constant [23], it still looks quite un-natural. In a word, if all of the datasets including the local determination of Hubble constant, Planck CMB data and BAO data are reliable, it should imply some new physics beyond the six-parameter base $\Lambda \mathrm{CDM}$ model. 
Acknowledgments We would like to thank Sai Wang for the helpful conversations. This work is supported by Top-Notch Young Talents Program of China, Grants from NSFC (Grant Nos. 11322545, 11335012 and 11575271) and a Grant from Chinese Academy of Sciences (No. QYZDJ-SSW-SYS006).

Open Access This article is distributed under the terms of the Creative Commons Attribution 4.0 International License (http://creativecomm ons.org/licenses/by/4.0/), which permits unrestricted use, distribution, and reproduction in any medium, provided you give appropriate credit to the original author(s) and the source, provide a link to the Creative Commons license, and indicate if changes were made. Funded by SCOAP ${ }^{3}$.

\section{References}

1. A.G. Riess et al. arXiv:1604.01424 [astro-ph.CO]

2. A.G. Riess et al., Astrophys. J. 730, 119 (2011). doi:10. 1088/0004-637X/732/2/129, doi:10.1088/0004-637X/730/2/119. arXiv:1103.2976 [astro-ph.CO]. [Erratum: Astrophys. J. 732, 129 (2011)]

3. G. Efstathiou, Mon. Not. Roy. Astron. Soc. 440(2), 1138 (2014). doi:10.1093/mnras/stu278. arXiv:1311.3461 [astro-ph.CO]

4. P.A.R. Ade et al. [Planck Collaboration]. arXiv:1502.01589 [astroph.CO]

5. C.L. Bennett et al. [WMAP Collaboration], Astrophys. J. Suppl. 208, 20 (2013). doi:10.1088/0067-0049/208/2/20. arXiv:1212.5225 [astro-ph.CO]

6. G. Hinshaw et al. [WMAP Collaboration], Astrophys. J. Suppl. 208, 19 (2013). doi:10.1088/0067-0049/208/2/19. arXiv:1212.5226 [astro-ph.CO]

7. C.L. Bennett, D. Larson, J.L. Weiland, G. Hinshaw, Astrophys. J. 794, 135 (2014). doi:10.1088/0004-637X/794/2/135. arXiv:1406.1718 [astro-ph.CO]

8. C. Cheng, Q.G. Huang, Sci. China Phys. Mech. Astron. 58(9), 599801 (2015). doi:10.1007/s11433-015-5684-5. arXiv:1409.6119 [astro-ph.CO]
9. P.A.R. Ade et al. [Planck Collaboration], Astron. Astrophys. 571, A16 (2014). doi:10.1051/0004-6361/201321591. arXiv:1303.5076 [astro-ph.CO]

10. K.T. Mehta, A.J. Cuesta, X. Xu, D.J. Eisenstein, N. Padmanabhan, Mon. Not. Roy. Astron. Soc. 427, 2168 (2012). doi:10.1111/ j.1365-2966.2012.21112.x. arXiv:1202.0092 [astro-ph.CO]

11. C. Cheng, Q.G. Huang, Phys. Rev. D 89(4), 043003 (2014). doi:10. 1103/PhysRevD.89.043003. arXiv:1306.4091 [astro-ph.CO]

12. E. Di Valentino, A. Melchiorri, J. Silk. arXiv:1606.00634 [astroph.CO]

13. M. Chevallier, D. Polarski, Int. J. Mod. Phys. D 10, 213 (2001). doi:10.1142/S0218271801000822. arXiv:gr-qc/0009008

14. E.V. Linder, Phys. Rev. Lett. 90, 091301 (2003). doi:10.1103/ PhysRevLett.90.091301. arXiv:astro-ph/0208512

15. Q.G. Huang, M. Li, X.D. Li, S. Wang, Phys. Rev. D 80, 083515 (2009). arXiv:0905.0797 [astro-ph.CO]

16. X.D. Li, S. Li, S. Wang, W.S. Zhang, Q.G. Huang, M. Li, JCAP 1107, 011 (2011). arXiv:1106.4116 [astro-ph.CO]

17. Q.G. Huang, K. Wang, S. Wang, JCAP 1512(12), 022 (2015). doi:10.1088/1475-7516/2015/12/022. arXiv:1509.00969 [astroph.CO]

18. F. Beutler et al., Mon. Not. Roy. Astron. Soc. 416, 3017 (2011). doi:10.1111/j.1365-2966.2011.19250.x. arXiv:1106.3366 [astroph.CO]

19. A.J. Ross, L. Samushia, C. Howlett, W.J. Percival, A. Burden, M. Manera, Mon. Not. Roy. Astron. Soc. 449(1), 835 (2015). doi:10.1093/mnras/stv154, doi:10.1093/mnras/stu523. arXiv:1409.3242 [astro-ph.CO], arXiv:1312.4877 [astro-ph.CO]

20. A.J. Cuesta et al. arXiv:1509.06371 [astro-ph.CO]

21. N. Aghanim et al. [Planck Collaboration]. arXiv:1605.02985 [astro-ph.CO]

22. R. Adam et al. [Planck Collaboration]. arXiv:1605.03507 [astroph.CO]

23. V. Marra, L. Amendola, I. Sawicki, W. Valkenburg, Phys. Rev. Lett. 110(24), 241305 (2013). doi:10.1103/PhysRevLett.110.241305. arXiv:1303.3121 [astro-ph.CO] 